\title{
Single-pixel imaging with Fourier filtering: application to vision through scattering media
}

\author{
Y. Jauregui-Sánchez ${ }^{1, *}$, P. Clemente ${ }^{1,2}$, J. Lancis ${ }^{1}$, and E. Tajahuerce ${ }^{1}$ \\ ${ }^{1}$ GROC·UJI, Institute of New Imaging Technologies (INIT), Universitat Jaume I, 12071, Castelló, \\ Spain \\ ${ }^{2}$ Servei Central d'Instrumentació Científica (SCIC), Universitat Jaume I, 12071, Castelló, Spain \\ ${ }^{*}$ Corresponding author: jauregui@uji.es
}

\begin{abstract}
We present a novel approach for imaging through scattering media that combines the principles of Fourier spatial filtering and single-pixel imaging. We compare the performance of our single-pixel imaging setup with that of a conventional system. First, we show that a single-pixel camera does not reduce the frequency content of the object, when a small pinhole is used as a low-pass filter at the detection side. Second, we show that the introduction of Fourier gating improves the contrast of imaging through scattering media in both optical systems. We conclude that single-pixel imaging fits better than conventional imaging on imaging through scattering media by the Fourier gating.
\end{abstract}

Keywords: Single-pixel imaging; Structured illumination; Spatial light modulators; Image through scattering media; Fourier spatial filtering; Turbid media.

https://doi.org/10.1364/OL . 44.000679

Imaging through scattering media has been a longstanding issue in many applications in engineering and biomedical imaging. Different approaches to tackle the problem can be classified as a function of the regime of light used to reconstruct the image $[1,2]$. Diffuse optical techniques such as diffuse optical tomography (DOT) rely on totally diffused photons. In this macroscopic regime, when light has traveled several transport mean free paths (TMFP), images are obtained by solving inverse problems based on diffuse models [3]. Recent techniques based on the control of light wavefronts with spatial light modulators (SLM) have allowed to develop imaging methods in an intermediate regime of light where photons are only slightly dispersed. These techniques enable us to work in a mesoscopic regime, reaching an intermediate depth range in addition to a good resolution $[4,5]$. Finally, some techniques for optical imaging through scattering media extract information of hidden objects from unscattered ballistic light. These ballistic techniques provide the best resolution although, of course, paying the price of a low penetration depth. Because, in general, the scattered component of the light is stronger than the ballistic component, these techniques aim to reduce the scattered one to improve the signal-to-noise ratio (SNR) of the final image. For instance, time-gating techniques select ballistic photons of light pulses by taking into account that they 
arrive earlier to the detector [6]. Other procedures to discriminate ballistic photons against scattered light are based on the use of some form of spatial filtering, by considering that most photons are scattered to higher spatial frequencies [2]. Fourier filtering is a practical and effective technique that, combined with ultrafast time-gating imaging, has allowed reduction of the scattered light by 10 orders of magnitude [7]. Recently, high-contrast images through scattering media have been obtained employing both Fourier filtering and structured illumination [8]. However, the use of a spatial filter to reduce the scattered light limits the achievable spatial resolution of the final image because high frequencies are blocked. In fact, there is a tradeoff between image resolution and contrast inherent to this technique. In this work we show how this tradeoff can be overcome by combining Fourier filtering and single-pixel imaging methods.

The first single-pixel imaging (SPI) techniques were described already in 1970 [9]. However, efficient cameras based on SPI were developed only recently [10] by using fast programmable spatial light modulators (SLM). SPI techniques are characterized by using structured illumination and bucket detection. The object is sequentially sampled with a set of microstructured light patterns codified onto an SLM, for instance a digital micromirror device (DMD). The light transmitted (or reflected) by the object is recorded by a single photosensor such as a photodiode or a photomultiplier tube (PMT). The image is reconstructed in the computer from the electric signal digitized by a data acquisition system (DAQ). Different algorithms can be used, such as a linear superposition, a change of basis, or a correlation operation. SPI has proved to be an effective technique for imaging through scattering media [11-13].

In this work, we describe an optical system working by transmission that combines SPI and the principles of Fourier spatial filtering to recover the image of an object hidden behind a turbid medium. We compare the performance of our optical setup with that of a conventional imaging system based on a CMOS camera. Our results show that introducing Fourier spatial filtering at the detection side improves the contrast of the images in both cases. However, the resolution of the conventional imaging system decreases with the amount of spatial frequency gating, while in the case of SPI the resolution loss is negligible. We conclude that SPI fits better than conventional imaging in vision through scattering media by Fourier filtering.

The experimental configuration to study the performance of the single-pixel camera (SPC) with Fourier filtering is shown in Figure 1(a). The microstructured light patterns are generated with a DMD illuminated with a monochromatic collimated light beam. An optical system in a 4 -f configuration, constituted by lenses L1 and L2, projects the patterns onto the object. A circular diaphragm is used to filter unwanted diffracted orders produced by the periodic micromirror arrangement on the DMD. Light is collected by lens L3 and focused onto a PMT. A pinhole with variable diameter located at the back Fourier plane of L3 performs the spatial frequency gating. With the aim of comparing, we use a second experimental setup constituted by a conventional 4 -f imaging system, formed by lenses L3 and L4, and a digital camera as is shown in Figure 1(b). Specifically, the light beam is generated with an incoherent white-light source (HPLS200, Thorlabs), and an interference filter (P10-515S 93819, Corion) with a bandwidth of 20 $\mathrm{nm}$ centered at $520 \mathrm{~nm}$. The DMD (DLP Discovery 4100, Texas Instrument) is a chipset array constituted by $1920 \times 1080$ micromirrors with a pixel pitch of $10.8 \mu \mathrm{m}$. The patterns codified on the DMD are $2 \mathrm{D}$ functions of the orthonormal Walsh-Hadamard (WH) basis [14]. Due to the binary modulation nature of the DMD, each WH function is projected combining two patterns, one codifying the positive and another the negative component of the function. These functions are projected onto the object at a frame rate of $20 \mathrm{~Hz}$. The focal distances of L1, L2 and L3 are the same, $f_{1}=f_{2}=f_{3}=100 \mathrm{~mm}$, while that of L4 is $f_{4}=75 \mathrm{~mm}$. The detector in Figure 1(a) is a PMT (PMM01, Thorlabs Inc.) and the sensor in Figure 1 (b) is a CMOS camera (UI-1540SE-M-GL, IDS) with $1280 \times 1024$ pixels with a pitch of $5.2 \mu \mathrm{m}$. The magnification factor of the 4-f optical system in Figure 1 (b) is 0.75 . The object in both experiments is a sector star target R1L1S2P that contains 36 radial sector pairs.

To operate with the SPC obtaining the maximum resolution and a wide field of view (FOV) in the shortest 
possible measurement time, we adopt the following strategy. We project different sets of microstructured patterns, all of them with a low number of pixels but with different sizes, adapting the spatial resolution of the sampling patterns to that of the object. Our approach is related with supersampling methods that use signal processing techniques to obtain highresolution images from multiple low-resolution samples [15]. It resembles the technique used in [16] for SPI but avoiding special foveated patterns. In particular, we project four sets of Hadamard patterns each one consisting of $64 \times 64$ pixels codifying the full collection of $4096 \mathrm{WH}$ functions for these resolution as is shown in Figure 2(a). Each pixel of the pattern is codified with a number of micromirrors that varies from $8 \times 8$, for the set with lower spatial resolution, to $1 \times 1$, for the patterns with higher spatial resolution. By projecting these patterns onto the object, and reconstructing the images with SPI techniques, we obtain four elemental images with $512 \times 512,256 \times 256$,
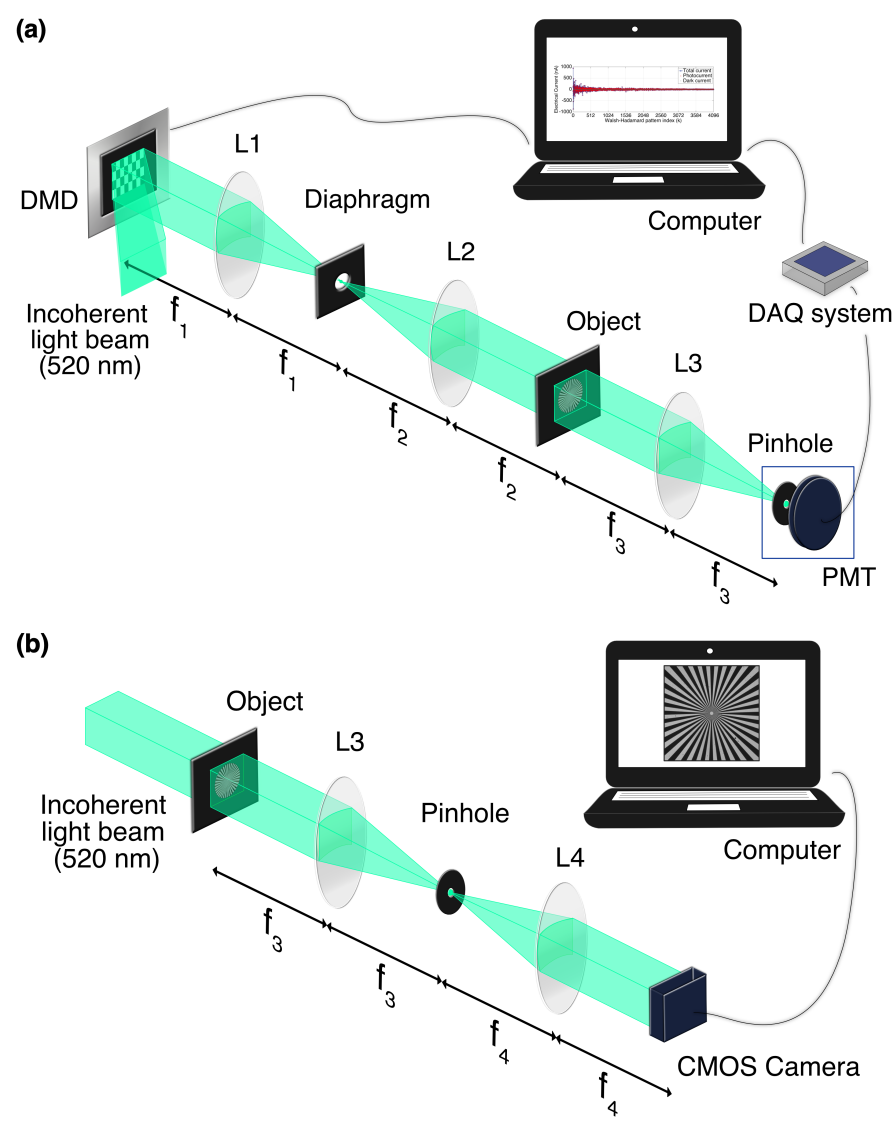

Figure 1: Schematic diagram of (a) the single-pixel camera and (b) the conventional imaging setup.
$128 \times 128$, and $64 \times 64$ pixels [Figure $2(\mathrm{~b})$ ]. The final image of the object is generated by combining the previous images into a single one, overlapping digitally the images with higher resolution over those with lower resolution, as is shown in Figure 2(d). Using this strategy, we recover an image with $512 \times 512$ pixels, with the maximum spatial resolution at the center, where the object has the smaller details, and lower spatial resolution at the borders. The number of projected patterns and, accordingly, the measurement time, is reduced by a factor of 16 , equal to the ratio of $512 \times 512$ to $4 \times 64 \times 64$. In our case, the reconstruction time is $\sim 14 \mathrm{~min}(\sim 0.8 \mathrm{~s}$ by using a fast DMD working at $20 \mathrm{kHz}$ ). Of course, the method is adjusted to the sector star target, but it will be welladapted also to other symmetric objects. In Figure 2(e) we plot the Michelson contrast, or visibility, of the four elemental images in Figure 2(b) as a function of the spatial frequency. It is obtained following the method described in Ref. [8]. For each elemental image, the threshold of the spatial frequency, which is marked with a vertical color line for each curve in Figure 2(e), corresponds to the pixel size of the specific WH pattern codified on the DMD. For our SPC, the highest spatial frequency is 46.29 line-pairs $/ \mathrm{mm}$. The solid black curve in Figure 2(e) shows the contrast of the final image in Figure 2(d). This is the curve used later for different filtering conditions in Figures 3(b) and 4(b).

To analyze the influence of Fourier filtering on SPI and compare it with the case of conventional imaging, we use a pinhole with variable diameter at the back Fourier plane of lens L3 in both optical systems in Figure 1. The experimental results are shown in Figure 3. Images in the first row of Figure 3(a) were obtained with the SPC, while those in the second row were recorded with the conventional one. Images on each column correspond to different Fourier filtering conditions: no pinhole for the first column and pinholes with a diameter of 2.0, 1.0, 0.3, and $0.2 \mathrm{~mm}$ for the subsequent columns, respectively. We see that the loss in resolution is negligible when performing Fourier spatial filtering in the SPI approach at detection side, even for small pinhole diameters. On the contrary, the loss is quite noticeable in the conventional imaging system as the pinhole diameter decreases. These experimental results are corroborated 
(a)

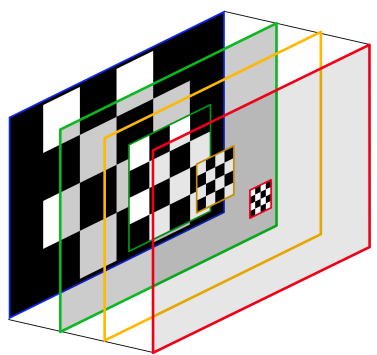

(b)

(c)

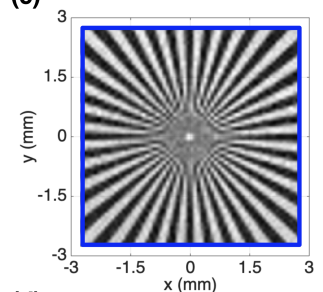

(d)

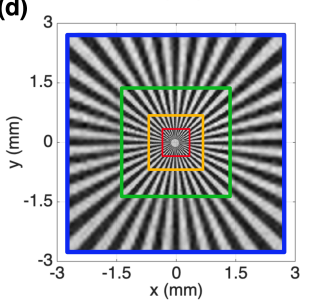

(e)

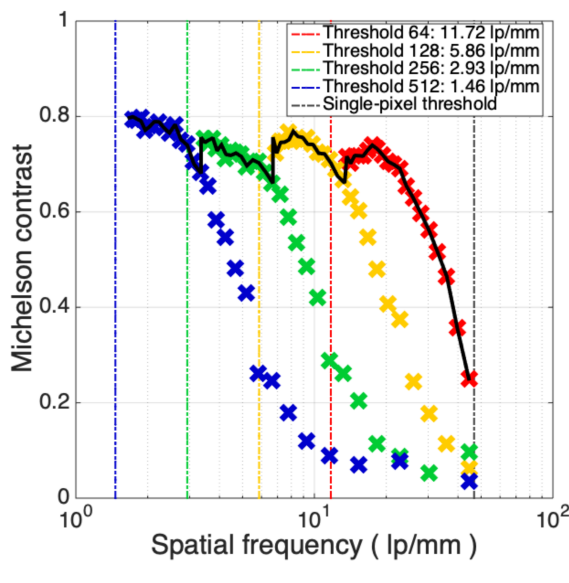

Figure 2: Experimental results obtained using the singlepixel camera setup: (a) Hadamard patterns with $64 \times 64$ pixels of different size codified on the DMD with different number of micromirrors; (b) Images of the sector star target with different spatial resolution recovered with the patterns in (a); (c) Low resolution image with $512 \times 512$ pixels [image marked blue in (b)]; (d) Final image, with $512 \times 512$ pixels, obtained by digital combination of the images in (b); (e) Michelson contrast as a function of the spatial frequency for each image in (b). The black envelope curve shows the contrast of the final image in (d).

by plotting the Michelson contrast as a function of the spatial frequency. Figures 3(b) and 3(c) show the results for the SPC and the conventional camera, respectively. Note that, by reducing the pinhole diameter, the contrast remains approximately constant for the case of SPI while it decreases significantly for the conventional imaging system. This behavior of the SPC is due to the characteristics of the measurement process. In SPI, the image is reconstructed by measuring the projection of the object onto a set of test functions, in our case functions of the WH basis. The key point is that this information is already present in the zero order of the Fourier transform of the product of the sampling patterns with the object. Note that the zero order provides the average of the

function being transformed, as can be verified from the expression of the Fourier transform with spatial frequencies equal to zero. Therefore, the object is still obtained by a simple linear superposition of the functions of the basis with the measured coefficient, even if we filter all frequencies except the zero order at the Fourier plane of the detection side of the camera. The only effect of the filtering process is a reduction of the light power efficiency. The result will be different by doing an equivalent low-pass filtering in the illumination side of the SPC, but this is not the case of our study.

A relevant application of Fourier filtering is on imaging through scattering media. It has been used to reject scattered light, thus increasing the proportion of ballistic light and, consequently, the image contrast $[7,8]$. However, this technique may also degrade the image quality, because the spatial-frequency gating operation will reduce also the spatial-frequency bandwidth of the final image. In view of the results obtained in the previous experiment, we propose to combine SPI techniques with Fourier spatial filtering to increase the quality of images of an object obtained through a turbid layer in a transmission configuration. To study the validity of the approach, we locate a scattering layer against the object in the SPI setup in Figure 1(a). The influence of the location of the scattering medium in SPI has been widely discussed in Refs. $[11,12,17]$. It has been proved that a turbid medium located between the object and the sensor, at the detection side, has no influence on the quality of the image. However, if the turbid medium is located between the DMD and the object, at the illumination side, the light patterns projected onto the object are degraded by scattering, and the quality of the final image decreases. Therefore, we choose the worse condition for SPI and locate the scattering layer at the illumination side. Again, we use a pinhole with variable diameter at the back Fourier plane of lens L3, now to filter the diffused photons and detect only the ballistic ones. For comparison, we repeat the experiment with the conventional system in Figure 1(b). In this case, the scattering layer is located at the detection side, as in Ref. [8]. Note that by applying the Helmholtz reciprocity principle of reversibility of light, both cameras are equivalent locating the scattering layer at different sides of the 
object. However, the introduction of the filter at the detection side, which is necessary to filter the scattered photons, breaks the symmetry and makes the SPC work better than conventional one, as we show next.

The experimental results are shown in Figure 4. As a turbid medium, we use epoxy resin with $\mathrm{TiO}_{2}$ ru-

(a)

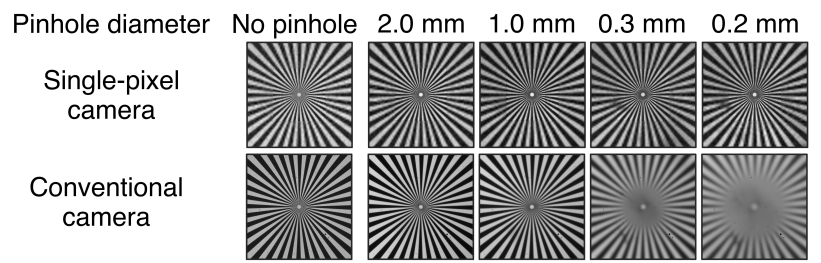

(b)

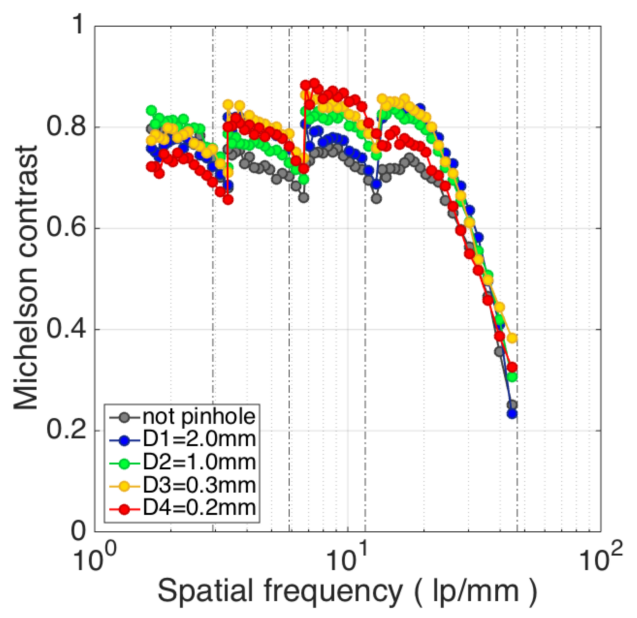

(c)

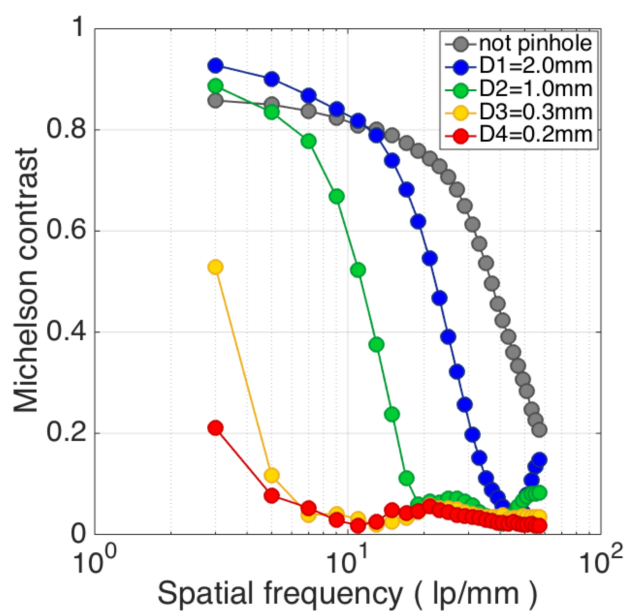

Figure 3: Experimental results without turbid medium. (a) Images obtained by both cameras and different Fourier filtering conditions. Michelson contrast versus spatial frequency for (b) the single-pixel camera and (c) the conventional optical imaging system. tile powder. A layer with a thickness of $3.27 \mathrm{~mm}$ was made following the recipe in Ref. [18]. We mixed $0.725(\mathrm{~g} / \mathrm{l})$ of $\mathrm{TiO}_{2}$ rutile powder (Titanium (IV) oxide, rutile powder, $<5 \mu \mathrm{m}$ particle size, Sigma Aldrich) with a 1:2 ratio of hardener (component B) to epoxy resin (component A). The different images were obtained in the same conditions as those in Figure 3 but now with the scattering layer. In both cam-

(a)

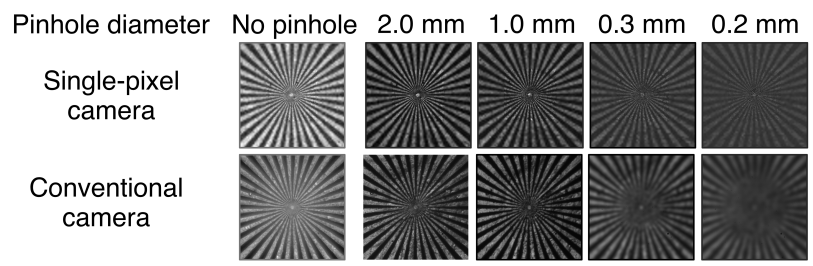

(b)

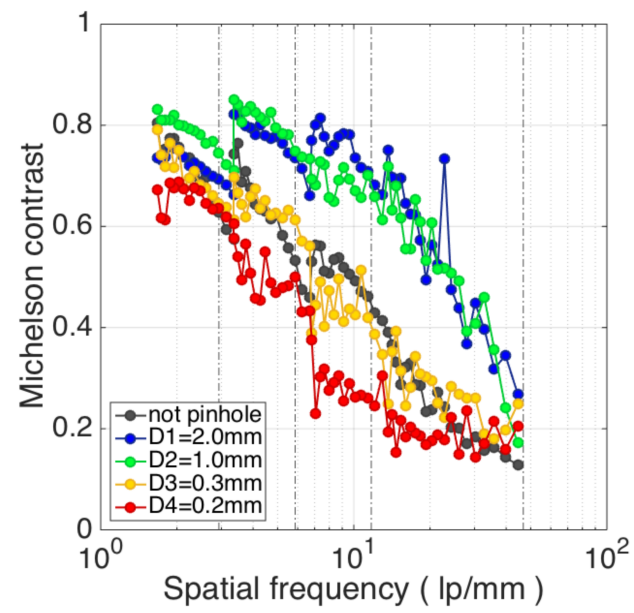

(c)

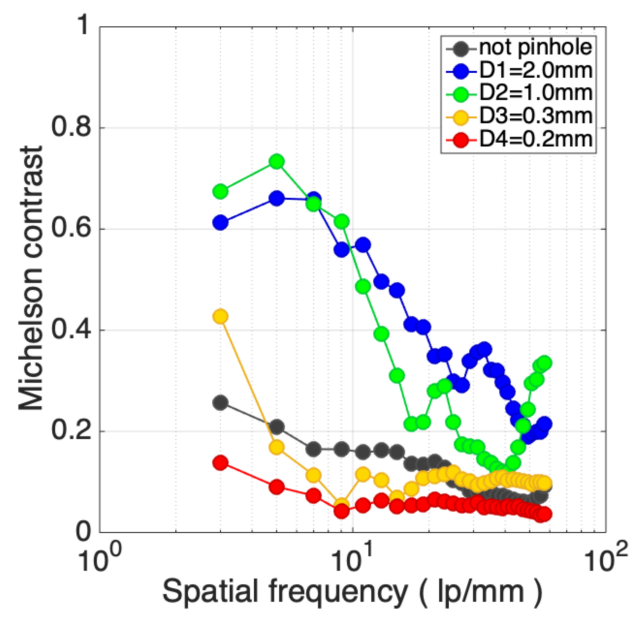

Figure 4: Experimental results with turbid medium. (a) Images obtained by both cameras with different Fourier filtering conditions. Michelson contrast versus spatial frequency for (b) the single-pixel camera and (c) the conventional optical imaging system. 
eras, the introduction of Fourier filtering improves the contrast of the images, as seen by comparing the result in the second column with those in the first one in Figure 4(a). This effect is corroborated by evaluating the Michelson contrast as a function of the spatial frequency of the images provided by both cameras. The curves are shown in Figures 4(b) and 4(c) for the case of the SPI system and the conventional one, respectively. Note that, in both cases, the resolution of the system worsens as the pinhole diameter decreases further. In the case of the conventional imaging system, the main reason is the low-pass filtering effect of the spatial frequencies, as was shown in Figure 3. In the case of SPI, the image resolution deteriorates because the SNR of the photocurrent provided by the PMT decreases for small diameters of the pinhole. However, the contrast of the images provided by the SPI system is better than that of the conventional one for all Fourier filtering conditions. For the case of no pinhole, the contrast is better because the SPC provides an inherent filtering effect. Highly scattered photons generate a uniform background pattern onto the object, instead of the well-defined Hadamard pattern generated by the ballistic photons, which is eliminated when the positive and the complementary signals provided by the two patterns codifying each WH function are subtracted in the reconstruction process of the image.

In conclusion, we have developed an imaging system combining SPI techniques with Fourier spatial filtering. We have compared the performance of our optical setup with that of a conventional imaging system based on Fourier filtering and using a CMOS camera as a detector. We have shown that, without scattering media, the single-pixel camera does not reduce the high frequency content of the object, when a small pinhole is used as a low-pass filter at the detection side. Moreover, when the scattering media are introduced, the Fourier spatial filtering technique improves the contrast of the images in both cases, the singlepixel camera and the conventional one. We conclude that SPI fits better than conventional imaging in vision through turbid media by Fourier filtering. We note that this effect is present in many SPI configurations using a photosensor with a reduced size. Therefore, it may contribute to improve the image quality in other applications of SPI in scattering media.
Funding. Ministerio de Economía y Competitividad (MINECO) (FIS2015-72872-EXP, FIS2016-75618-R); Generalitat Valenciana (PROMETEO/2016/079); Universitat Jaume I (UJI) (P1-1B2015-35).

Acknowledgment. Y. Jauregui-Sánchez acknowledges financial support from Generalitat Valenciana (GRISOLIA/2015/037).

\section{References}

[1] V. Ntziachristos, "Going deeper than microscopy: the optical imaging frontier in biology," Nat. Meth. 7, 8603-8614 (2010).

[2] C. Dunsby, and P. M. W. French, "Techniques for depth-resolved imaging through turbid media including coherence-gated imaging," J. Phys. D. Appl. Phys. 36, R207-R227 (2003).

[3] D. A. Boas, D. H. Brooks, E. L. Miller, C. A. DiMarzio, M. Kilmer, R. J. Gaudette, and Q. Zhang, "Imaging the body with diffuse optical tomography," IEEE Signal Proc. Magazine, 18, 57-75 (2001).

[4] S. Gigan, "Optical microscopy aims deep," Nat. Photonics 11, 14-16 (2017).

[5] A. P. Mosk, A. Lagendijk, G. Lerosey, and M. Fink, "Controlling waves in space and time for imaging and focusing in complex media," Nat. Photonics 6, 283-292 (2012).

[6] L. Wang, P. P. Ho, C. Liu, G. Zhang, and R. R. Alfano, "Ballistic 2-D imaging through scattering walls using an ultrafast optical Kerr gate," Science 253, 769-771 (1991).

[7] Q. Z. Wang, X. Liang, L. Wang, P. P. Ho, and R. R. Alfano, "Fourier spatial filter acts as a temporal gate for light propagating through a turbid medium," Opt. Lett. 20, 1498-1500 (1995).

[8] E. Berrocal, S. G. Pettersson, and E. Kristensson, "High-contrast imaging through scattering media using structured illumination and Fourier filtering," Opt. Lett. 41, 5612-5615 (2016). 
[9] J. A. Decker, Jr., "Hadamard-transform image scanning," Appl. Opt. 9, 1392-1395 (1970).

[10] M. F. Duarte, M. A. Davenport, D. Takhar, J. N. Laska, T. Sun, K. F. Kelly, and R. G. Baraniuk, "Single-pixel imaging via compressive sampling," IEEE Signal Process. Mag. 25, 83-91 (2008).

[11] E. Tajahuerce, V. Durán, P. Clemente, E. Irles, F. Soldevila, P. Andrés, and J. Lancis, "Image transmission through dynamic scattering media by single-pixel photodetection," Opt. Express 22, 16945-16955 (2014).

[12] V. Durán, F. Soldevila, E. Irles, P. Clemente, E. Tajahuerce, P. Andrés, and J. Lancis, "Compressive imaging in scattering media," Opt. Express 23, 14424-14433 (2015).

[13] Y. K. Xu, W. T. Liu, E. F. Zhang, Q. Li, H. Y. Dai, and P. X. Chen, "Is ghost imaging intrinsically more powerful against scattering?," Opt. Express 23, 32993-33000 (2015).

[14] W. K. Pratt, J. Kane, and H. C. Andrews, "Hadamard transform image coding," Proc. IEEE 57, 58-68 (1969).
[15] S. C. Park, M. K. Park, and M. G. Kang, "Super-resolution image reconstruction: a technical overview," IEEE Signal Process. Mag. 20, 21-36 (2003).

[16] D. B. Phillips, M. J. Sun, J. M. Taylor, M. P. Edgar, S. M. Barnett, G. M. Gibson, and M. J. Padgett, "Adaptive foveated single-pixel imaging with dynamic supersampling," Sci. Adv. 3, 1-10 (2017).

[17] A. D. Rodríguez, P. Clemente, E. Irles, E. Tajahuerce, and J. Lancis, "Resolution analysis in computational imaging with patterned illumination and bucket detection," Opt. Lett. 39, 38883891 (2014).

[18] Y. Jauregui-Sánchez, P. Clemente, J. Lancis, and E. Tajahuerce, "Imaging through scattering media by Fourier filtering and single-pixel detection", Proc. SPIE 10502, Adaptive Optics and Wavefront Control for Biological Systems IV, 105020W1-105020W6 (2018). 